\title{
The neuralgic amyotrophy consultation
}

Received: 10 March 2006

Received in revised form: 31 March 2006

Accepted: 12 April 2006

Published online: 20 April 2007
N. van Alfen, $\mathrm{MD}, \operatorname{PhD}(\square)$

Neurologist, clinical neurophysiologist

Neuromuscular Centre Nijmegen

Dept. of Neurology and Clinical

Neurophysiology

c/o $920 \mathrm{KNF}$

Radboud University Nijmegen

Medical Centre

PO Box 9101

6500 HB Nijmegen

The Netherlands

Tel.: +31-24/2613491

Fax: +31-24/3615097

E-Mail: n.vanalfen@neuro.umcn.nl
Abstract Neuralgic amyotrophy is a distinct clinical syndrome with acute severe pain and patchy paresis in the shoulder and arm region. The clinical phenotype was recently found to be more comprehensive and the long-term prognosis less optimistic than usually assumed for many patients. The disorder can be idiopathic or hereditary in an autosomal dominant fashion, with only few phenotypical variations between the two. This article provides a practical overview of current knowledge on the clinical presentation, diagnosis, pathogenesis and the treatment of pain and complications.
Key words brachial plexus . differential diagnosis . neuralgic amyotrophy · treatment

\section{Introduction}

Neuralgic amyotrophy (NA) is a distinct clinical entity [1]. Core features are the extreme neuropathic pain at onset and the rapid development of multifocal pareses and atrophy, usually in the upper extremity, as well as the slow recovery in months to years. Sensory signs or symptoms are usually not very marked, although on careful examination patchy hypaesthesia can be found in most patients. In the majority of patients, the right shoulder is affected, and the tell-tale sign of NA for most physicians is the conspicuous winging of the shoulder blade, the scapula alata, although this is present in only two-thirds of the patients. Especially in the hereditary form, but sometimes also in extensive attacks of idiopathic NA, other peripheral nerves outside the brachial plexus can also be involved, such as lower cranial nerves or the lumbosacral plexus. Painless attacks occasionally, occur and are only recognized as NA because of a sudden patchy peripheral paresis in the upper extremities without any identifiable cause.

Neuralgic amyotrophy has both an idiopathic (INA) and hereditary form (HNA). HNA (OMIM 162100) is autosomal dominant with a high but not complete penetrance estimated at $80 \%$. In the idiopathic form, patients usually suffer only one attack in their life, but up to $25 \%$ may go on to suffer a recurrence. In HNA attacks recur more frequently, in 
almost $75 \%$ of the patients. [1] NA is more common in men (male to female ratio $3: 2$ ), with an age of onset in INA usually in the second or third decade but ranging from the neonatal age to the seventh decade. Patients with HNA tend to be younger when their first attack occurs, usually in their $2^{\text {nd }}$ decade. Although neuralgic amyotrophy is considered a rare disease, the minimum incidence of idiopathic NA is estimated at $2-4 / 10^{5}$ /year, making the disorder as common as the Guillain-Barré syndrome or primary malignant CNS tumours $[5,15]$. The hereditary form is much rarer, with about 200 families known worldwide to date.

In the authors' 10-year experience NA is still relatively unknown to many physicians, even though the clinical picture is usually typical. The initial diagnosis is very often thought to be shoulder joint pathology or cervical radiculopathy. Patients with NA are usually first seen by a family physician and referred to neurologists and/or orthopedic surgeons, but may be even more frequently referred to physical therapists at first or subsequently, especially in the initial phase when the diagnosis has not yet been established. One of the major problems in diagnosing neuralgic amyotrophy is that the course and localisation of both pain and paresis can vary considerably among patients, and even among recurrent attacks in one patient. [1] Sometimes the only signs of an attack are annoying pain in the upper arm lasting a few hours with a subsequent loss of pinch grip for a few months, caused by a lesion of the anterior interosseus nerve. Conversely, the patient may have suffered from severe pains in both shoulders and arms for weeks on end, has a serious orthopnoea requiring nocturnal positive pressure ventilation, and cannot use either of his or her arms or hands in daily life for several years because of severe bilateral plexus damage with involvement of both phrenic nerves.

This review aims to provide the neurological practitioner with an update on the clinical features, diagnosis and pathogenesis of neuralgic amyotrophy and a practice-based overview of the current diagnostic and treatment options. Reported numbers and percentages are derived from a recent cohort study [1] unless noted otherwise.

\section{Clinical presentation}

\section{Pain types}

An acute onset of an unusually severe and relentless pain in the neck, shoulder and / or arm regions is very characteristic of the disorder and occurs in $96 \%$ of all patients. It often $(61 \%)$ wakes patients in the middle of the night or early morning, and usually increases to maximum severity in a few hours. Then it has almost invariably become very severe, and unlike anything the patient experienced before (unless he or she had a previous attack). Instructing the patient to grade the pain on a scale of 0 to 10 ( 0 being no pain and 10 the most severe pain imaginable) is an illustrative way of making this clear, because initially $60 \%$ and subsequently $90 \%$ of the patients will have such a NRS (Numerical Rating Scale) score of 7 or more, and the median NRS is 8 at onset and 9 at maximum intensity. The pain can also have a stuttering onset, and it can take up to two weeks or more for paresis to develop. Pain is usually worse at night and $94 \%$ of the patients cannot sleep because of it. Typically attempts to mitigate it by assuming certain postures or using acetaminophen or NSAIDs provide no relief whatsoever, and patients often tell that the pain had them up all night sitting on the couch with their arm pressed tightly against their body, or even had them crawling desperately on the floor not knowing what to do anymore. It is a story not lightly forgotten once heard, and the next patient will probably be recognized by it too, making it a useful diagnostic tool.

Once the acute stage of an attack is over, i.e. the initial pain has subsided and a patchy paresis and atrophy have become evident, $77 \%$ of the patients will go on to suffer from one or two additional types of pain. First the damaged nerves in the plexus can give rise to an increased mechanical sensitivity, eliciting shooting or radiating neuropathic pain in the affected nerves' territory by putting strain on them through extension, abduction or elevation of the arm (a "Lasegue sign of the arm"). This hypersensitivity usually dissipates after weeks to months. Additionally many patients also develop musculoskeletal-type pain localized to the origin or insertion of the paretic - or compensating - muscles, especially in the periscapular, cervical and occipital regions. This pain may be particularly therapy-resistant and can sometimes become almost as incapacitating as the residual paresis itself. The main risk for developing it seem to be probably scapular instability caused by a paresis of the serratus anterior, rhomboids or trapezius. One the one hand this can lead to local myalgia and muscle strain in the trapezius and levator scapulae if they have to compensate for a downward and laterally displaced scapula. Often it is maximally felt at the points where these muscles attach to the occiput, cervical spine or shoulder blade, and can radiate further to the forehead - resembling tension-type headache - or interscapular region. Myalgia and pain at the attachment sites frequently also arises in paretic periscapular muscles that (also) serve a postural function. It is most often found in the serratus anterior region, extending from the medial scapular edge via the subscapular region and axilla to the midaxillary chest wall where the muscle inserts onto the ribs. 
This pain can be so bothersome that it even prevents normal breathing, because chest wall movement will further increase it. Obviously this complication can be even more impairing when the patient also has concomitant phrenic nerve dysfunction. Besides in the serratus anterior region it may occur in the area of any paretic muscle in NA. And finally, pain can result from glenohumeral joint pathology such as rotator cuff impingement or capsular irritation, developing as a consequence of the altered shoulder biomechanics in NA (see below).

\section{Motor and sensory symptoms}

The second typical feature of NA, that can at the same time make it difficult to recognize by clinicians, is the patchiness of the motor and sensory symptoms. Histological studies have already shown that the pathologic, presumably inflammatory, process can cause very focal damage to one or a few of the fascicles that make up a brachial plexus trunk or cord or a peripheral nerve, while simultaneously scatteredly affecting several parts of the plexus as a whole $[2,12]$. This is reflected clinically by a wide variety in the possible distribution - and severity - of paresis and sensory deficits. Any part of the brachial plexus, and clinically any muscle or skin area can be involved, in all sorts of combinations. Sensory symptoms or pain tend not to correlate with the localisation of the paresis. [1] It is precisely the recognition of this patchiness that is a very important clue to the diagnosis of the plexopathy in NA.

There are several overall patterns of paresis that occur more frequently than others. An upper brachial plexus distribution - the periscapular and perihumeral muscles - is most common, and occurs in $71 \%$ of the patients, either with $(50 \%)$ or without $(21 \%)$ involvement of the long thoracic nerve that leads to an unstable or winged scapula. Women have symptoms in a middle or lower brachial plexus distribution twice as often $(23 \%)$ as men (11\%), including symptoms that mainly affect the interosseus anterior nerve, with reduced pinch grip strength due to weakness of the long thumb and index finger flexors. Almost $80 \%$ of the patients have sensory deficits on examination, and NA can also present with pain and sensory symptoms only, frequently in the distribution of the lateral cutaneous antebrachial nerve [18]. In 15\% of the attacks there are signs of distal autonomic nervous system dysfunction (e.g. hand oedema, vasomotor instability). Additionally, nerves outside the distribution of the brachial plexus can also be involved, and this occurs in 56\% of the attacks in HNA patients and in $17 \%$ of the attacks in the idiopathic form. They usually involve the lumbosacral plexus (usually in a lumbar trunk distribution), phrenic nerve or recurrent laryngeal nerve, but occasionally more 'exotic' structures such as the facial or abdominal nerves may be affected too.

One of the main pitfalls in the diagnostic phase is that the patients tend to complain about that part of their shoulder or arm that is most impaired by either pain or paresis, but hardly notice or give attention to other, lesser impairments in strength or sensory loss. About a third of the attacks have bilateral involvement that usually is asymmetrical in its severity, so here too one side can easily get overlooked. As a clinician one should be aware of this, and supplement the history by a meticulous physical examination that specifically pays attention to those muscles or skin areas the patient doesn't mention. Omitting this increases the chance of either diagnostic errors (e.g. making the clinical diagnosis of a mononeuropathy or radiculopathy instead of a plexus lesion) or difficulty in correctly explaining certain symptoms, such as mistaking an unstable and downwardly displaced scapula for atrophy of the trapezius muscle, or its subsequent inability to move the arm overhead for a deltoid paresis.

\section{Shoulder biomechanics}

For most clinicians the biomechanics of shoulder and arm movements are not part of daily practice. However, a little knowledge about the anatomical relations between the scapula, chest wall and humerus will greatly facilitate the understanding of why damage to certain parts of the plexus or peripheral nerves gives rise to impaired movements or joint complications that cannot be explained by paresis only.

It is important to realize that the scapula, through its glenoid cavity and acromial extension, forms half of the glenohumeral joint, i.e. the socket. [20] For fluent movements of the arm it is necessary that the other half, the humeral head, remains approximately centered in its socket during the whole range of motion. This is normally achieved through two simultaneous mechanisms: rotation of the scapula to optimize the position of the glenoid cavity, and the action of several muscles that lift, depress or rotate the humeral head during abduction to ensure its optimal position (Figs. 1 and 2; reproduced with kind permission of K. van Nugteren from "Orthopedische Casuïstiek"). These two mechanisms must be able to work in a well-coordinated fashion because otherwise the humeral head will become impinged against the acromion, initially compressing elements of the interposed rotator cuff and subsequently making further movement mechanically impossible due to collision of both joint surfaces. The rotator cuff is 


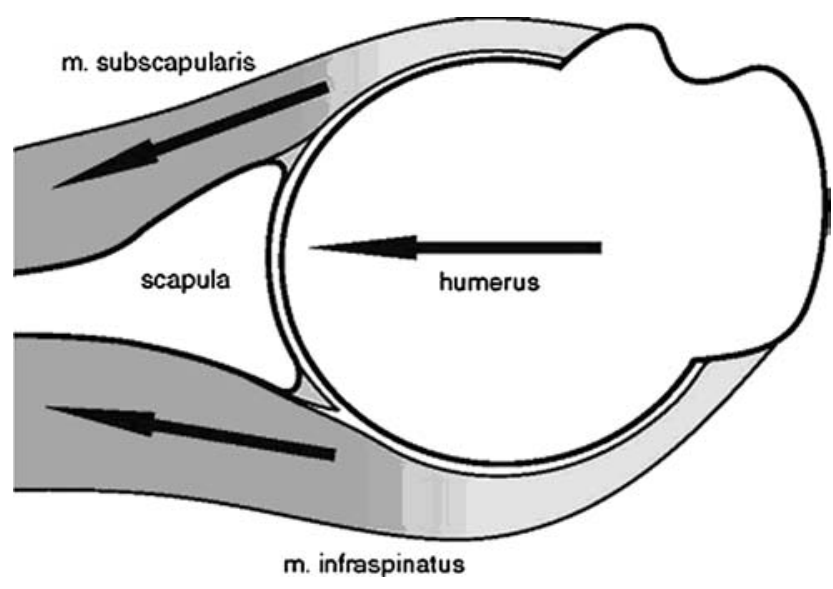

Fig. 1 Transversal plane schematic of muscles keeping the humeral position optimized against the scapula

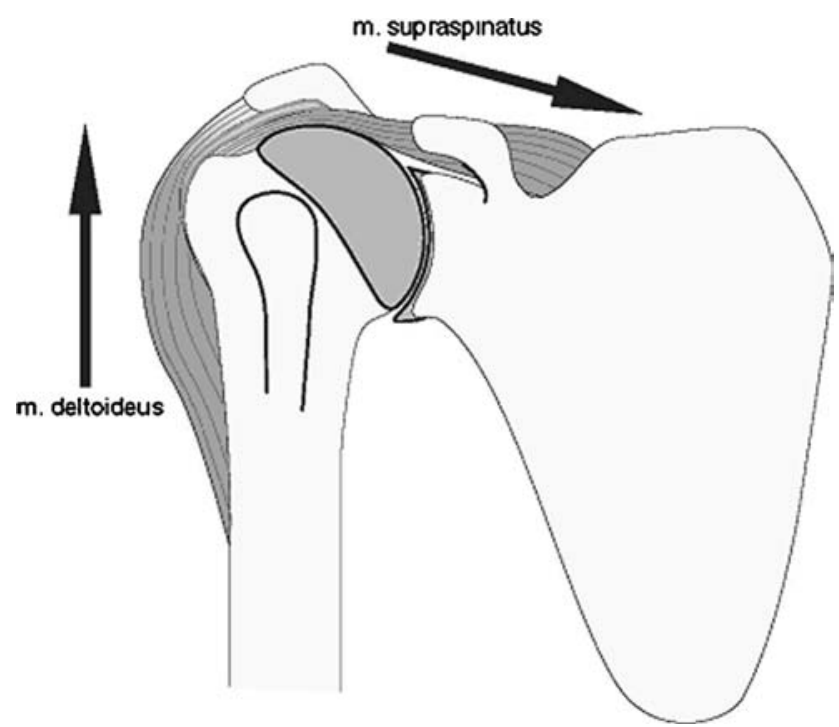

Fig. 2 Coronal plane schematic of muscles that keeping the humeral position optimized against the scapula

made up of the brachii supraspinatus, subscapularis, teres minor and biceps tendons. At rest, the humeral head is also kept in place by the action of the deltoid muscle. Weakness < MRC 3 of this muscle can lead to sagging of the humeral head, creating a dynamic subluxation that depends on the residual strength and fatigue of this muscle. Vice versa if the deltoid is unaffected but the other cuff muscles are weak it can also lead to a dynamic impingement because of unopposed cranial elevation of the humeral head during deltoid contraction (Fig. 2). The downward and lateral displacement of the scapula on the chest wall in case of serratus weakness also leads to an altered position of the glenoid and acromion at rest which makes the humeral head protrude - or subluxate - anteriorly, increasing the chance of impingement.

For fluent abduction of the arm at a level of more than $80^{\circ}$ elevation scapular support of the humeral head is indispensable. [16] The arm has to be tilted up and outward by contraction of the supraspinatus and deltoid, which can only be fully achieved when the rotated humeral head is supported from below by the scapular socket to be moved further up and out. This means that when scapular movement is impaired, e.g. when the serratus anterior is $<$ MRC 3 , abduction and elevation will automatically be impaired above this level, even when the deltoid supplying the force for this movement is intact. When in doubt whether insufficient scapular movement or paresis of the deltoid is responsible for decreased arm abduction, one can attempt to fixate the scapula by compressing it against the chest while exerting counterpressure with the other hand placed underneath the patients arm anteriorly on the chest. In a case of pure serratus weakness the patient should now be able to lift the arm more easily to maximally $110^{\circ}$ (to complete this motion to $170^{\circ}$ elevation scapular movement is required).

The periscapular - serratus anterior, trapezius, rhomboid - muscles are not just important for abduction and elevation of the arm. They also serve an important postural function in stabilizing the scapula to provide a fixed support for any arm and hand movements in front of or behind the trunk. In this capacity they are activated in any body posture except lying supine. This means that even sitting or walking will cause symptoms when these muscles are paretic, especially when the arms own weight cannot be supported. When scapular instability and impaired motion is present patients often subconsciously attempt to compensate and increase stability by adducting the shoulder through contraction of the trapezius, in addition with lateroflexion of the trunk to the contralateral side to elevate the affected shoulder further and facilitate abduction by helping tilt the humeral head anteriorly. Although in itself at least partially effective, this strategy frequently leads to myalgia in the trapezius and paraspinal muscles due to strain.

\section{Physical examination and pitfalls}

Because NA attacks frequently involve the proximal or upper parts of the brachial plexus and therefore often influences shoulder biomechanics, there are some issues that merit special attention during the physical examination. It is convenient to start out with inspection of the shoulder blades, assessing their respective position on the chest wall. In case of 
serratus anterior or - much less frequently - trapezius weakness the shoulder blade sags a few centimeters downward (because of the weight of the arm attached) and in serratus palsy is usually also laterally displaced with the lower border medially rotated at rest. Winging, or a scapula alata, can be observed at rest but becomes more prominent with arm movement. If the affected shoulder appears to be higher than the unaffected side it is usually because the patient is compensating the downward scapular sagging by tensing the trapezius muscle; after testing the following relaxation will often show the downward displacement at rest.

In case of severe serratus anterior weakness the whole shoulder and arm can slide off the chest wall to a more caudal and anterior position. If one is unaware of this type of paresis, it can easily be mistaken for atrophy of the trapezius muscle because the edge of the shoulder is lower on that side. Sometimes we found the aberrant position of the scapula in combination with supraspinatus atrophy had given rise to the impression that a tumour protruded from the shoulder blade, while in fact it was the crest of the scapular spine.

Besides testing all the individual muscles or muscle groups for strength grading, it is also useful to inspect the so-called scapulothoracic and glenohumeral rhythm in NA patients. The first can be done by having the patient slowly abduct and elevate the arm in a coronal plane to the maximum of 170 degrees and then moving it downward in the sagittal plane, and vice versa. The normal scapular motion outwards and inwards during this movement should be fluent, but when impaired it becomes jerky, indicating weakness of the serratus, or less often also the rhomboids or trapezius. To test the glenohumeral joint for impingement, the patients flexed arm can gently be lifted to maximum abduction in the coronal plane by the examiner while keeping the other hand on the acromion and humeral head; there should be no crepitation, restriction of movement or local pain in part of the arc and the humeral head should not luxate (if necessary keep it fixed with your fingers to prevent this). Restricted capsular movement can be tested by passive arm exorotation, comparing the range of excursions to that of the contralateral side. During the previous tests it is also possible to note any mechanical or stretch-sensitivity of the affected nerves (a "Lasegue sign of the arm").

As many patients experience musculoskeletal-type pain in both affected and compensating muscles in the months to years following an attack, which can sometimes be difficult to differentiate from the neuropathic pains occurring in NA, it is useful to differentiate these paintypes by palpating the bulk and attachments of these muscles to detect atrophy, hypertrophy and tenderness that points to strain. The levator scapulae (a part of which can be palpated just anterior to the trapezius at the point where the neck changes into the shoulder), its insertion on the occiput and scapular crest, and the insertion of the pectoralis minor on the coracoid process are often especially painful when the serratus anterior is weak.

Another pitfall we came across was the impression of deltoid or biceps weakness in cases with scapular instability. To avoid this influencing strength grading in these muscles, one may test the deltoid at about 70 - 80 degrees lateral elevation, and have the patient firmly adduct the flexed elbow against the chest for resistance testing the of biceps, thereby limiting the role of scapular stability in these movements.

Because the phrenic nerve is involved in 7\% (INA) to $14 \%$ (HNA) of the patients it is also useful to inspect the diaphragm for paradoxical breathing, i.e. the in- and upward movement of the abdominal wall during deep inspiration instead of the normal downand outward displacement. When present this is strongly suggestive of (hemi-) diaphragmatic paralysis, and this could be confirmed by having the patient bend over or lie down supine to see if there is orthopnoea (which occurs due to even further restriction of the lung volume by upward movement of the abdominal contents in these positions).

As sensory deficits in NA are often restricted to relatively small skin areas, it is useful to compare pinprick sensation of both sides in both shoulders, arms and hands. Proprioceptic loss is very rare in NA, but one can find signs of vasomotor instability indicating focal autonomic system dysfunction in the forearm and hands of some $15 \%$ of the patients.

\section{Diagnosis}

Once heard of or seen before, neuralgic amyotrophy is a very distinct clinical syndrome in $95 \%$ of the cases [1], that can usually be recognized from the patients history only. There are very few if any disorders in the shoulder or arm region that are so extremely and non-abatingly painful at onset, and once the patchy paresis or atrophy sets in the diagnosis should be readily apparent. A simple three-step way to diagnose $\mathrm{NA}$ and differentiate it from its most common differential diagnostic entities would be to start with the following questions: is the pain acute, very severe (NRS score $>7$ ) and unlike anything the patient had before? If yes, NA is likely, if not NA is possible but one should consider alternative diagnoses. Is there a limitation of passive arm exorotation or abduction? If not, NA is likely, but if yes shoulder joint pathology (bursitis, tendinitis calcarea) is more likely. And 
thirdly, are all symptoms (pain, paresis and sensory disturbances) in the same root distribution? If not NA is likely, if yes a cervical radiculopathy is more likely.

\section{Differential diagnosis of acute upper extremity pain and paresis}

However, some disorders mimic the clinical picture of NA (Table 1). Cervical radiculopathies typically present with posture-dependent radiating pain and sensory disturbances, and sometimes paresis, in the corresponding dermatome. As they are usually caused by nerve root compression due to degenerative spinal column disease, they occur most frequently at the levels that suffer the largest amount of mechanical strain during life. The estimated incidence of these radiculopathies is highest at the C7 level (about 40/ $10^{5}$ /year), and decreases towards more cranial levels (C6: $14 / 10^{5}$ /year, C5: 5/10 /year). [17] When they present in an acute fashion it is likely that the cause is a disk rupture with herniation, which usually occurs at a single level at a time. If there is a progressive degenerative spondylosis with foraminal stenosis patients can suffer from radiculopathies at multiple levels simultaneously, but will present with gradually progressive symptoms.

A true multiple mononeuritis, i.e. a peripheral nervous system vasculitis, can give rise to multiple progressive, but mostly distal focal nerve lesions in the arms. As the chance of being affected by such a vasculitic process is highest in the longest axons, patients usually also suffer from focal neuropathies in the legs and will often progress to a polyneuropathylike distribution of their symptoms. [8] Multiple focal pareses in the arm can also be found in multifocal motor neuropathy, a patchy, painless, and pure motor inflammatory peripheral nervous system disorder with a predilection for the forearm. Sometimes a carpal tunnel syndrome can present atypically, with pain and paraesthesias radiating up the arm to the shoulder region. Rarely, a brachial plexopathy-like distribution is seen in a focal subtype of motor neuron disease called brachial amyotrophic diplegia. [11]

Of course non-neurological disorders, such as inflammatory or degenerative shoulder, elbow, or wrist joint disease also present with pain and limitations of upper extremity movements. 'Shoulder complaints' for example, are very common in the general population, with an estimated incidence of around $2000 / 10^{5} /$ year in the Netherlands. Careful examination will usually reveal the arthrogenic cause, and sensory symptoms or a real paresis are typically not seen with these disorders. It can be difficult to rule out a paresis clinically though, for example in cases with a biceps or supraspinatus tendon rupture resulting in an inability to convey muscular contraction strength to the joint, or movements resulting in increased pain that prevents any further attempts at motion. Correctly identifying these symptoms as nonneurological will require an adequate index of suspicion and appropriate testing from the examiner.

\section{Differential diagnosis of plexopathies}

When the clinical diagnosis of a brachial plexopathy has been made, the aetiological possibilities are usually limited (Table 2). Many plexopathies are either caused by direct trauma (e.g. a motor vehicle accident) or iatrogenic damage such as following irradiation or surgery. The lower brachial plexus lesion seen in patients with a Pancoast tumour has a distinct, ominous course, with progressive pain and sensory disturbances and eventually paresis, spreading from the $\mathrm{T} 2$ and $\mathrm{T} 1$ segments to more proximal sites as the tumour grows. A similar typical lower plexus distribution pattern, of a more benign but still disabling nature, can be seen in true neurogenic thoracic outlet syndrome (incidence: 1 per million), in which the lower trunk of the plexus containing the C8 and T1 root extensions becomes compressed by a fibrous band extending from an elongated $\mathrm{C} 7$ transverse process to the first rib. Typically the sensory disturbances are in the medial forearm and ulnar side of the hand, while motor symptoms and atrophy prevail in the thenar and to lesser extent in the hypothenar muscles. [10]

When no cause is apparent and the plexopathy had a rapid painful onset, the cause is most likely neuralgic amyotrophy. Rarely, a similar picture but usually with progressive symptoms can be found with direct peripheral nervous system infections such as neuroborreliosis or HIV. In painless idiopathic brachial plexopathies with only limited paresis and sensory symptoms it can sometimes take weeks to months before the patient notes anything is wrong. In such a case it can be difficult to exclude other insidious causes such as a nerve or nerve sheath tumour of the plexus. Even without additional investigations clinical follow-up will then usually tell them apart, by showing that the disorder is really progressive, as would be expected in case of a neoplasm, or slowly improving as it will in idiopathic cases. Rarely another seemingly idiopathic and sometimes also painful brachial plexopathy can be the presenting symptom in patients with a hereditary neuropathy with liability to pressure palsies (HNPP), in which a relatively minor stretch or compressive trauma can damage the vulnerable nerves.[7] These patients usually - but not always - have a (family) history of compressive neuropathies at other sites (e.g. carpal 
Table 1 Differential diagnosis of upper extremity pain and/or paresis

\begin{tabular}{ll}
\hline & Difference with NA \\
\hline $\begin{array}{l}\text { Neurological disorders } \\
\text { cervical radiculopathy, degenerative } \\
\text { cervical radiculopathy, disk rupture }\end{array}$ & $\begin{array}{l}\text { insidious onset, slowly progressive or fluctuating course } \\
\text { acute onset, pain varies with posture, pain, sensory and motor } \\
\text { symptoms in same dermatome } \\
\text { mononeuritis multiplex / PNS vasculitis } \\
\text { multifocal motor neuropathy } \\
\text { brachial amyotrophic diplegia }\end{array}$ \\
$\begin{array}{l}\text { Non-neurological disorders } \\
\text { shoulder or elbow joint pathology posture } \\
\text { painless, no sensory symptoms, distal predominance, progressive } \\
\text { insidious onset, no sensory symptoms painless, progressive }\end{array}$ \\
$\begin{array}{c}\text { cervical spondylosis with referred brachialgia } \\
\text { complex regional pain syndrome }\end{array}$ & $\begin{array}{c}\text { passive restriction of movement } \\
\text { often posture or activity dependent, no focal deficits, fluctuating course } \\
\text { vasomotor symptoms predominate diffuse pain and weakness subacute } \\
\text { onset with progression }\end{array}$ \\
\hline
\end{tabular}

Table 2 Differential diagnosis of plexopathy aetiologies

\begin{tabular}{ll}
\hline Aetiology & Difference with NA \\
\hline $\begin{array}{l}\text { Trauma } \\
\text { Postradiation }\end{array}$ & $\begin{array}{l}\text { direct relation in time with trauma, force direction predicts damage localisation } \\
\text { usually } 2-10 \text { years after radiotherapy, slowly progressive, prominent paraesthesias } \\
\text { lower trunk lesion, direct relation in time to surgery, resolves in weeks-months } \\
\text { painless wasting of thenar }>\text { hypothenar, slowly progressive, } \\
\text { hypaesthesia in medial forearm }\end{array}$ \\
$\begin{array}{l}\text { True neurogenic thoracic outlet syndrome } \\
\text { gradual onset, usually slowly progressive, symptoms vary with localisation } \\
\text { insidious onset, progressive pain, symptoms spread from lower plexus to } \\
\text { middle and upper parts; Horner's syndrome }\end{array}$ \\
$\begin{array}{l}\text { Pancoast tumour } \\
\text { often painless, can resolve rapidly, concomitant entrapment neuropathies, } \\
\text { polyneuropathy in elderly patients }\end{array}$ \\
$\begin{array}{l}\text { Hereditary neuropathy with liability } \\
\text { to pressure palsies }\end{array}$
\end{tabular}

tunnel syndrome or dropfoot), and signs of a polyneuropathy when they are older. HNPP can nowadays be confirmed both at the electrophysiological and DNA level.

\section{Ancillary investigations in NA}

Laboratory investigations can show some abnormalities in about $25 \%$ of the patients (signs of previous infection, mildly elevated CK, elevated liver enzymes probably related to preceding infection, antiganglioside antibodies, slightly increased CSF protein content), but usually these findings do directly not contribute to the diagnosis. In typical cases laboratory investigations are therefore unnecessary, but if patients are in any way at risk it can be wise to rule out a direct infectious cause for the plexopathy due to neuroborreliosis or HIV because the initial clinical picture can be identical.

Many clinicians will perform additional cervical spine imaging by MRI or CT scan, in search for or to rule out a cervical disk herniation or degenerative changes. When the clinical lesion is that of a pure monoradiculopathy with pain, sensory symptoms and paresis all in the same dermatome it is certainly rec- ommended as this combination of symptoms would also be unusual in NA. But one should bear in mind that a certain number of people have asymptomatic disk herniation, especially at the C6-C7 level, and also that the incidence of a cervical radiculopathy at the C5 level is equal to that of NA itself. [17] In more than half of otherwise typical NA cases degenerative changes of the cervical spine are found [1], and one should therefore be cautious about placing too much emphasis on the radiological rather than the clinical picture. MRI scanning of the brachial plexus can be warranted to rule out space-occupying lesions if the symptoms are progressive. In NA it will typically show no abnormalities, but in a small percentage T2 hyperintensity of affected plexus parts can be found.

Often chest radiography is performed to exclude an apical lung (Pancoast) tumour, although the chance of finding such a lesion is small when the patient has an upper trunk brachial plexopathy of acute onset. One advantage of this relatively simple investigation is that it simultaneously evaluates the position of the diaphragm halves, detecting a paresis in some $10 \%$ of NA patients. If such a paresis is already suspected on clinical grounds it is advisable to include an ultrasound examination of diaphragm movement or fluoroscopy, and a pulmonary function 
study comparing supine and sitting positions in the diagnostic investigations (a difference of $>400-500$ $\mathrm{ml}$ is abnormal and typical for diaphragmatic insufficiency).

An electrophysiological examination including electromyography (EMG) and nerve conduction studies (NCS) is generally the only ancillary investigation that can positively support the diagnosis of NA. [19] NCS can show reductions in the sensory nerve action potential amplitudes in different brachial plexus branches, excluding a solely radicular problem. Motor nerve conduction studies are usually not very informative unless the paresis is severe, in which case the compound motor action potentials will be reduced in affected nerves. It can also be helpful in evaluating the phrenic nerves. NCS can also help in making other diagnoses unlikely, such as HNPP or multifocal motor neuropathy in painless cases. Needle examination will identify neurogenic abnormalities in affected muscles, inform the examiner on the severity of the lesions, and can confirm that the involvement is in a multifocal plexus distribution when clinical doubt exists. Of course the same pitfall exists for the EMG as for the clinical examination: one has to know which affected muscles to study or otherwise the results will be false negative and the diagnosis missed. In the first years after an attack EMG can show the extent of sometimes clinically unnoticeable - reinnervation and recovery, making it an important tool in helping predict functional outcome.

\section{Pathogenesis}

The exact pathophysiological mechanism of NA is as yet unknown. The current hypothesis is that the attacks are caused by an immune-mediated response to one's own peripheral nerves. [19] One of the main indications that NA is an immune-mediated disorder came from the fact that about half of the attacks are preceded by an event that could have triggered the immune system, such as infections, surgery, pregnancy and the puerperium, 'stress' (both mental and strenuous physical exercise), immunisations, and immunodulating therapies with interleukin-2 or interferone-alfa2 [1, 6, 19]. However, two interesting reports of NA epidemics showed that in addition to a preceding infection mechanical factors or a specific genetic background may also be a prerequisite to trigger an attack. $[3,4]$ There are also patients who had no obvious immunological preceding event but described strenuous physical activity as a possible trigger. And since there are many patients (46.8\%) who have not noticed any preceding event whatsoever, neither infection nor mechanical stress seem to be able to explain the whole story of the onset of NA attacks.

Additionally, immune-mediated mechanisms may explain the occurrence of attacks, but do not yet tell us why one person is susceptible to NA while another is not. In patients with hereditary NA the genetic factor seems the most logical choice for an explanation of at least part of the underlying susceptibility. In the past decades a number of HNA families were found to harbour a genetic locus for the disorder on chromosome 17q25. In other families this could not be confirmed, implying that HNA is heterogeneous. [14] Recently three mutations in the SEPT9 gene were found in several 17q25-linked families by effort of an extensive international collaboration, although the role of these mutations is still speculative [13]. The recent finding that in INA patients the recurrence rate after a first episode is much higher (10-25\%) than the incidence of NA in the general population $\left(2-3 / 10^{6} /\right.$ year) [1] strongly implies that these patients too must have some sort of innate susceptibility to the attacks. In summary, current evidence suggests that neuralgic amyotrophy is a disorder with a complex pathophysiological mechanism in which autoimmune, genetic and external factors all seem to play an interwoven role.

\section{Management}

\section{Pain treatment}

How and if pain should be treated depends mainly on which stage of the attack the patient is in. As described the pain in NA comes in different phases and types: the acute neuropathic pain at onset, residual neuropathic and mechanical hypersensitivity pain next, and finally musculoskeletal pains in paretic and compensating muscles and sometimes the shoulder joint that can remain as long as recovery is incomplete. And although the initial pain is one of the most characteristic features of the disorder the impact of the subsequent pain types should not be underestimated, as about $1 / 3$ of the patients still suffer from these pains after an average follow-up of 6 years.

The acute phase pain has been found to respond best to a combination of a long-acting NSAID and opiate, such as diclofenac slow-release $100 \mathrm{mg}$ bid with slow-release morphine $10-30 \mathrm{mg}$ bid [1]. The sole use of an over-the-counter prescription such as acetaminophen or a NSAID as a rule does not provide adequate relief and should not be advised. Co-analgesics such as gabapentin, carbamazepine or amitriptyline may be helpful for the second phase pain, that is characterized by spontaneous or movementinduced shooting pains and tingling sensations due to 
aberrant impulse firing in the damaged, hypersensitive parts of the plexus. These are not really suited for use in the acute phase because of their delayed onset of effect, which usually lasts about as long (several weeks) as the acute phase pain itself.

\section{Physical therapy and rehabilitation}

Treatment of the third, musculoskeletal type of pain following an NA attack is much more of a challenge. In practice it has been found very resistant to pharmacological and even sophisticated (e.g. nerve block) pain treatment. The only effective intervention is helping the patient re-establish an optimized biomechanical situation. This can be done by a combination of physical therapy to maintain fluent periscapular motion and prevent dysfunctional compensating strategies, and well-timed alternation between rest and activities in daily life. Physical therapists can help with pain recognition and prevention, and instruct the patient how to maintain fluent scapulothoracic and glenohumeral motion. In addition, most therapists will attempt to optimize residual strength by certain exercises, and the overall impression is that patients who keep their affected limb active are better off functionally. There are a few pitfalls, however, when it comes to these strengthening exercises in NA. The most common therapy failure occurs when attempts are made to strengthen the intact compensating periscapular muscles whilst the serratus anterior is weak. Although the concept seems valid from a theoretical point of view, there are almost no exercises of this type that can be performed without concomitant serratus activation. This makes patients very prone to developing (additional) myalgia and fatigue in this muscle which will in turn keep them from further exercise. A similar pitfall is seen when muscles with strength below MRC grade 3 are trained using weights. Here one can safely assume that if a muscle is not able to lift the attached bodyweight against gravity it will certainly not be able to do so if extra kilos are added. And finally one can say that in general trying to train muscles that are already strained due to altered biomechanics will inherently carry a risk of additional strain and increasing symptoms of pain and fatigue.

The use of a foam rubber sling to support the weight of the arm when sitting, standing or walking relieving the scapula-stabilizing muscles of the arm weight - can be a useful part of this strategy, but one should ensure that at the same time the patient maintains full joint motion by passive or active movement a few times a day to prevent contractures. Quite often patients themselves are already applying this support 'trick' by tucking their arm and hand in a coat pocket when standing or walking. Commercially available scapular braces can also provide support, but have the disadvantage that they can only optimize one of the two periscapular muscles functions (postural or kinetic) at a time. To fixate the scapula against the thorax for maximum stability they have to be worn very tightly around the chest. This is both uncomfortable and may even restrict normal breathing excursion, and it prevents arm abduction above $110^{\circ}$ elevation because of lack of scapular movement. NA patients who found them supportive have usually worn them less tightly, but just tight enough to provide a little support for the arm weight during sitting and standing, similar to what a sling would do.

Treatment of glenohumeral joint pathology involves physical therapy and local injection with a combined anaesthetic and corticosteroid when capsular or tendon / cuff irritation or bursitis are present. In case of a partial cuff tendon rupture rest is advised; a complete rupture has to be surgically treated. It is also important to look for work or daily life-associated movement patterns that can maintain this type of complication (especially repetitive arm movements or excessive weight-bearing) and make the patient aware of this association. In severe cases where conservative management fails, it may be necessary to resort to orthopaedic surgical intervention, but it goes without saying that if possible one should aim to prevent this. Patients with phrenic nerve palsy and orthopnoea should be evaluated for nocturnal hypoventilation and REM-sleep apnoeas, and treated with positive pressure ventilation if required.

Since initially the severe pain will prevent many patients from attending their work, the inevitable question will arise when it is wise to return to work. The best answer to this question from the NA perspective would be 'at the earliest time possible', because there is no evidence that physical activities will prevent nerve recovery or provoke another attack. However, it is obvious that depending on the type of posture and the activities required certain jobs will increase the chance of complications described in the section on the third pain phase. A gradual increase of activities, with spreading of the amount of time spent in certain postures (e.g. sitting behind a desk, working with a keyboard, lifting objects) and alternating these physically demanding activities with other less demanding tasks will usually be preferable as long as weakness and impairment is significant. Fatigue was found to be a complication of NA attacks in over a quarter of the patients (unpublished observation) and seems to be part of the NA phenotype in some. Spreading one's activities over the day and choosing what to spend one's energy on and what not seems the most reasonable advice for these patients. For more specific help and guidance we offer patients the consultation of a specialized physiatrist as a part of standard care. 


\section{References}

1. van Alfen N, van Engelen BGM (2006) The clinical spectrum of neuralgic amyotrophy in 246 cases. Brain 129: 438-450 (Brain 2005 Advance access online publication Dec $21^{\text {st }}$, DOI 10.1093/brain/awh722)

2. van Alfen N, Gabreels-Festen AA, Ter Laak HJ, Arts WF, Gabreels FJ, van Engelen BG (2005) Histology of hereditary neuralgic amyotrophy. J Neurol Neurosurg Psychiatry 76:445-447

3. Auge WK 2nd, Velazquez PA (2000) Parsonage-Turner syndrome in the Native American Indian. J Shoulder Elbow Surg 9:99-103

4. Bardos V, Somodská V (1961) Epidemiologic study of a brachial plexus neuritis outbreak in northeast Czechoslovakia. World Neurol 2:973-979

5. Beghi E, Kurland LT, Mulder DW, Nicolosi A (1985) Brachial plexus neuropathy in the population of Rochester, Minnesota, 1970-1981. Ann Neurol 18:320-323

6. Bernsen PL, Wong Chung RE, Vingerhoets HM, Janssen JT (1988) Bilateral neuralgic amyotrophy induced by interferon treatment. Arch Neurol 45:449-451

7. Chance PF (1999) Overview of hereditary neuropathy with liability to pressure palsies. Ann N Y Acad Sci 883:1421
8. Collins MP, Periquet MI, Mendell JR, Sahenk Z, Nagaraja HN, Kissel JT (2003) Nonsystemic vasculitic neuropathy: insights from a clinical cohort. Neurology 61:623-630

9. Clarkson PM, Hubal MJ (2002) Exercise-induced muscle damage in humans. Am J Phys Med Rehabil 81(11 Suppl):S52-69

10. Huang JH, Zager EL (2004) Thoracic outlet syndrome. Neurosurgery 55:897903

11. Katz JS, Wolfe GI, Andersson PB, Saperstein DS, Elliott JL, Nations SP, Bryan WW, Barohn RJ (1999) Brachial amyotrophic diplegia: a slowly progressive motor neuron disorder. Neurology 53:1071-6

12. Klein CJ, Dyck PJB, Friedenberg SM, Burns TM, Windebank AJ, Dyck PJ (2002) Inflammation and neuropathic attacks in hereditary brachial plexus neuropathy. J Neurol Neurosurg Psychiatry 73:45-50

13. Kuhlenbaumer G, Hannibal MC, Nelis E, Schirmacher A, Verpoorten N, Meuleman J, Watts GD, Vriendt ED, Young P, Stogbauer F, Halfter H, Irobi J, Goossens D, Del-Favero J, Betz BG, Hor H, Kurlemann G, Bird TD, Airaksinen E, Mononen T, Serradell AP, Prats JM, Broeckhoven CV, Jonghe $\mathrm{PD}$, Timmerman $\mathrm{V}$, Ringelstein EB, Chance PF (2005) Mutations in SEPT9 cause hereditary neuralgic amyotrophy. Nat Genet 37:1044-1046
14. Kuhlenbaumer G, Meuleman J, De Jonghe P, Falck B, Young P, Hunermund G, Van Broeckhoven C, Timmerman V, Stogbauer F (2001) Hereditary Neuralgic Amyotrophy (HNA) is genetically heterogeneous. J Neurol 248:861-865

15. MacDonald BK, Cockerell OC, Sander JW, Shorvon SD (2000) The incidence and lifetime prevalence of neurological disorders in a prospective community-based study in the UK. Brain 123:665-676

16. Mottram SL (1997) Dynamic stability of the scapula. Man Ther 2:123-131

17. Radhakrishnan K, Litchy WJ, O’Fallon WM, Kurland LT (1994) Epidemiology of cervical radiculopathy. A population-based study from Rochester, Minnesota, 1976 through 1990. Brain 117:325-335

18. Seror P (2004) Isolated sensory manifestations in neuralgic amyotrophy: report of eight cases. Muscle Nerve 29:134-138

19. Suarez GA (2005) Immune brachial plexus neuropathy. In: Dyck PJ, Thomas PK, (eds). Peripheral neuropathy. Elsevier Saunders, Philadelphia, pp 2299-2308

20. Terry GC, Chopp TM (2000) Functional Anatomy of the Shoulder. J Athl Train $35: 248-255$ 